\title{
Arbitrary digital pulse sequence generator with delay-loop timing
}

\author{
Radim Hošák ${ }^{1}$ and Miroslav Ježek, ${ }^{1}$ ) \\ Department of Optics, Faculty of Science, Palacký University, 17. listopadu 12, 77146 Olomouc, \\ Czech Republic
}

(Dated: January 9, 2018)

\begin{abstract}
We propose an idea of an electronic multi-channel arbitrary digital sequence generator with temporal granularity equal to a single clock cycle. We implement the generator with 32 channels using a low-cost ARM microcontroller and demonstrate its capability to produce temporal delays ranging from tens of nanoseconds to hundreds of seconds, with 12 ns timing granularity and linear scaling of delay with respect to the number of delay loop iterations. The generator is optionally synchronized with an external clock source to provide 100 ps jitter and overall sequence repeatability within the whole temporal range. The generator is fully programmable and able to produce digital sequences of high complexity. The concept of the generator can be implemented using different microcontrollers and applied for controlling of various optical, atomic, and nuclear physics measurement setups.
\end{abstract}

\section{INTRODUCTION}

An arbitrary digital pulse sequence generator is a crucial tool for many instrumentation, automatization, and metrological tasks. It is frequently used to control and synchronize other devices to form highly complex setups like magnetic resonance imaging, trapped ions experiments ${ }^{2 / 3}$, and fast reconfigurable photonic circuits ${ }^{4}$ for quantum simulations and quantum communication networks 5 , to name a few applications. A large number of electronic and optoelectronic building blocks such as switches, digital attenuators, direct digital synthesizers, digital-to-analog converters, electro-optic or acoustooptic modulators, and gated solid-state detectors, which are employed in these setups, require many digital control channels with precise timing. Commercial solutions typically offer limited flexibility and impractically large resources per channel. Arbitrary waveform generators (AWGs) and digital delay generators (DDGs) represent two examples of the frequently used commercial devices. AWGs allow high degree of sequence control, which goes far beyond a digital pattern, but are severely limited in sequence length. They are very inefficient in producing long sparse sequences. DDGs offer picosecond timing and long delays but are unable to generate complex sequences with many changes of the output signal.

The lack of flexible digital generators with reasonable timing properties and a large enough number of output channels leads to the development of custom-built solutions based on prototyping platforms, such as microcontrollers (MCUs) ${ }^{7 / 9}$ and field programmable gate arrays (FPGAs) 10 . The custom solutions often also offer analog and radio frequency signal\$911. MCUs have gained significant computing power, they can be easily programmed, and their large number of peripherals makes them highly flexible, the downside of which is that the reaching of full temporal control down to single clock cycle represents a challenging task. FPGAs offer a single cycle or even sub-cycle resolution employing a serialization approach 10 . However, the development on this

a)Electronic mail: jezek@optics.upol.cz platform is more complicated compared to the MCUs, particularly the asynchronous design represents a challenging task.

The design of a custom digital pulse sequence generator, further called pulsebox, typically utilizes one of three basic approaches: (1) timer peripherals, (2) recalling the sequence from a fast memory, and (3) communicating the sequence during a runtime. Using timers to clock delays between the sequence events $7 / 9$ offers good temporal resolution but the corresponding interrupt overhead impacts negatively the minimum time interval between the events. The fast memory approach allows for singleclock sequence programming, however, it causes a strict resolution vs. sequence length trade-off as digital states of all channels for every clock cycle have to be saved in the memory. This approach is particularly suitable for short sequences with high temporal resolution 12 13. Alternatively, the digital states of the pulsebox can be programmed during its runtime using external communication, which imposes no limitation on the sequence complexity 10. However, ongoing communication overhead limits the minimum time interval between the sequence events and makes such the solution particularly suitable for long and sparse sequences.

The presented work focuses on a custom pulsebox device which produces digital signals on many output channels. The output signals take on the form of arbitrary pulse sequences, such as the one in Fig. 1. The sequences may contain both rapid successions of short pulses and pulses with great temporal separation. It is desirable that both these features be available within the single sequence generated by the pulsebox. We propose here a concept of this flexible pulsebox, its implementation using an ARM microcontroller, and a set of characteristics along with methods for their measurement.

\section{DESIGN}

A pulse sequence can be understood as a number of digital output changes occurring at specified times. The pulsebox can reproduce any pulse sequence, as long as for each output change two pieces of information are known: the desired digital state of all output channels after the 
output change, and the time at which the output change is to occur. Our design relies on a 32bit microcontroller unit (MCU) whose digital pins are divided into ports of 32 and individually tied to the pulsebox digital outputs, either directly or via auxiliary circuitry such as level shifters or buffer amplifiers. The states of the 32 individual digital pins of each port are governed by a 32-bit register. Digital pins belonging to the same port are used so that the state of all the pulsebox output channels can be changed synchronously by manipulating a single register. The 16 randomly selected channels were characterized during the calibration stage to demonstrate correct operation. One digital pin of the MCU is used as an input for an external trigger signal.

To correctly reproduce the pulse sequence, the output changes need to occur at the specified times. Without artificially introduced delay, two consecutive output changes will be separated by a time interval corresponding to the time it takes the MCU to modify the port register. If the output changes need to be more separated in time, it is necessary to produce an extra delay by instructing the MCU to stay idle using the NOP assembly instruction 14 . This performs a no-operation which takes one clock cycle. By repeating this instruction in the source code, the distance between two output changes can be changed by the unit of one clock cycle. This is the best granularity possible for an MCU-based pulsebox.

Using the NOP instruction yields very good granularity, but is not desirable for long delays, as the MCU program memory would fill up very quickly. Instead, the NOP instruction is looped over inside a delay loop. The loop can be repeated almost infinitely, making a very large temporal distance between consecutive output changes possible. However, the loop consists of multiple instructions. This means that the granularity is worse than when using NOP instructions exclusively.

The delay loop consists of two distinct parts - the setup and the looped section. The setup specifies the number of loop iterations and its processing by the MCU takes a constant amount of time. The looped section is repeated a given amount of times and is responsible for most of the delay achieved via the delay loop. The time spent in the delay loop setup gives a lower bound on the length of the shortest pulse achievable using this method, and the length of the looped section determines the granularity with which we can vary the delay lengths. The loop has thus been designed to minimize the number of instructions in both the setup and the looped section, leading us to implement it in assembly language.

To access the entire range of achievable temporal distances between consecutive output changes, and still benefit from the one-cycle granularity, both the NOP approach and the delay loop approach must be combined. The only drawback here comes from the way the MCU predicts which instructions will be performed next. When conditional branches occur, which is the case for the delay loops, the program execution might stall up to three clock cycles before the MCU discovers which instructions follow. This could lead to an up to three-clock

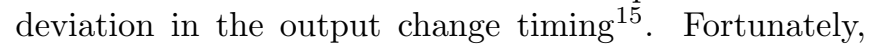
this is a systematic deviation and not a jitter, as the timing stays constant throughout sequence repetitions.

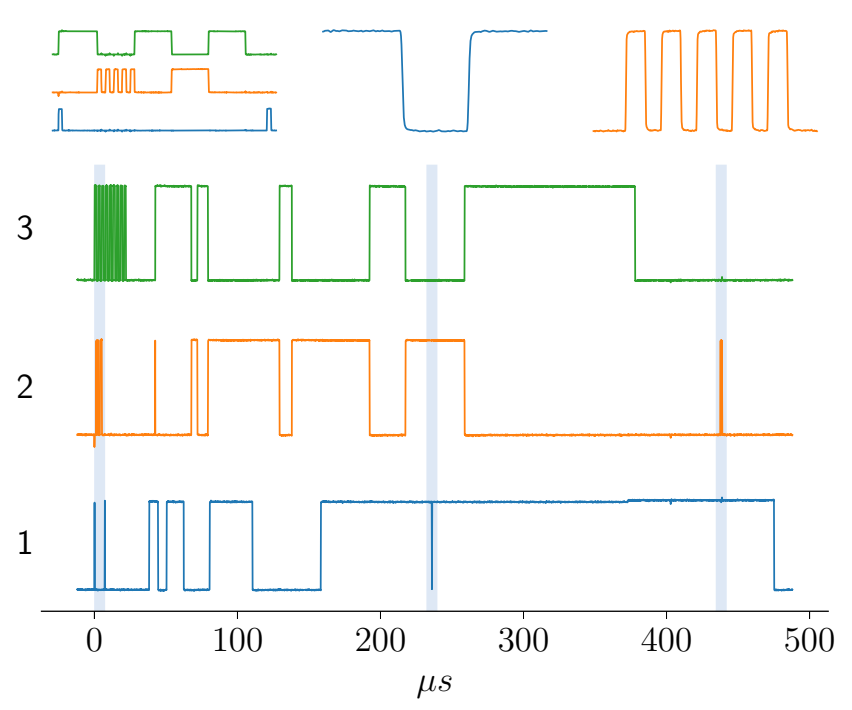

Figure 1. An example of a complex three-channel pulse sequence. The sequence pattern consists of both rapid successions of pulses and output changes which occur sparsely in time. The highlighted regions near the beginning, midpoint, and ending of the sequence are shown in greater detail. The shortest structure takes $120 \mathrm{~ns}$, the whole sequence has a duration of $0.5 \mathrm{~ms}$. The depicted waveforms are the actual output of a prototype of the presented pulsebox device.

This effect can be eliminated by a detailed calibration that would provide feedback for the sequence input.

The final part of the design is the software package for user-friendly reconfiguration of the device. The software gathers user-provided data about the shape of the sequence, generates a source code file with instructions for the pulsebox to reproduce the sequence, compiles it, and uploads the resulting binary file to the MCU. The software is able to extract the sequence shape data from different user-provided information, such as the positions and lengths of individual pulses. The source code is created by gluing together instructions for changes of states of the MCU digital pins with delay loops that provide the MCU with idle time between output changes.

Our physical realization of the concept mentioned above relies on the Arduino Due development board based on the Atmel SAM3X8E microcontroller $\frac{16}{16}$ Arduino Due has been previously used for custom-built control solutions in several advanced applications including optical tweezers, preparation and measurement of nonclassical states of light, and quantum memory 17 19. Implementing the pulsebox on this low-cost development board demonstrates the feasibility of the delay-loop concept and, also, further extends the applicability of similar MCUs for advanced analog as well as digital control.

\section{PULSEBOX PERFORMANCE}

To characterize the performance and capabilities of the device, we have chosen these figures of merit: (1) the minimum and (2) maximum time interval between output changes, (3) timing granularity, (4) linearity, (5) run-to- 


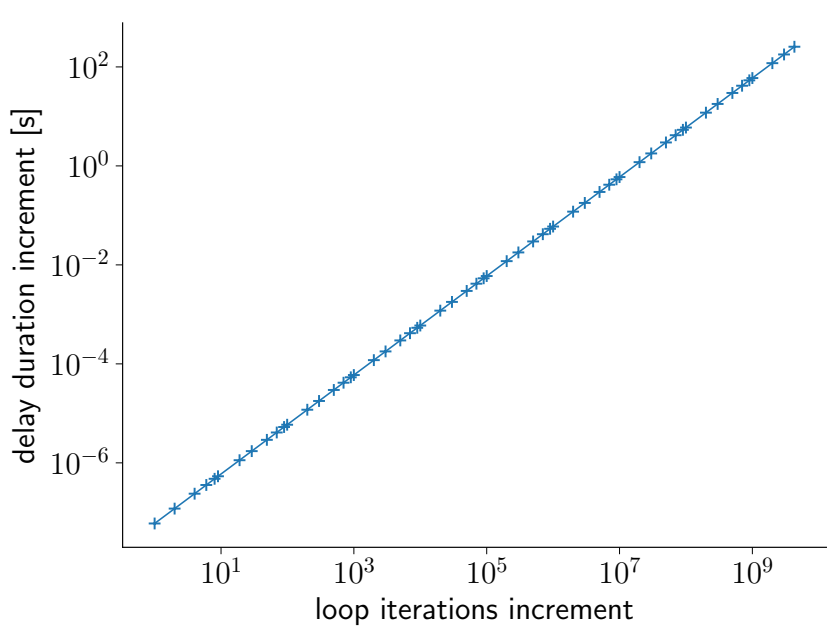

Figure 2. The delay duration increment is shown as a function of the increment in a number of delay-loop iterations. The calibration data are fitted to a linear function. See text for more details.

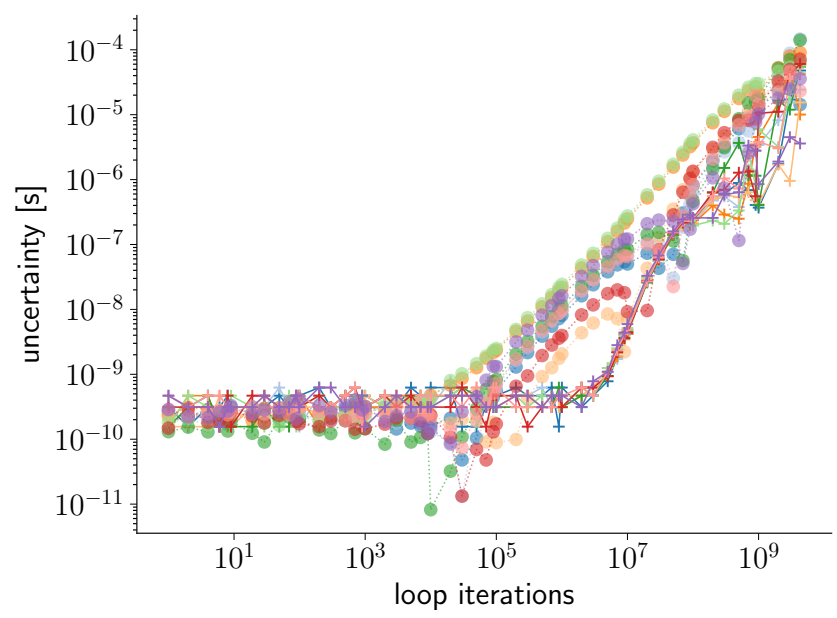

Figure 3. The delay duration span (cross marks, solid lines) and the absolute value of the mismatch between the data and the calibration line fit (circle marks, dotted lines) are shown for each calibration run. An increase in both the delay span and the fit mismatch, caused by the drift and/or instability of the internal clock, is seen after $10^{5}$ iterations.

run uncertainty, (6) inter-channel simultaneity, (7) trigger latency, and (8) maximum sequence complexity.

To measure the minimum time interval between output changes, an output change, and then immediately another one, were made on a single channel, resulting in a single pulse. The minimum pulse length was then the time interval between the $50 \%$ crossings of the rising and falling edge of the pulse, which we measured using an oscilloscope. For our device, the result was 24 ns, which corresponds to two clock cycles of the MCU. Using the delay loop approach, the minimum pulse length was achieved using a single iteration loop and was measured to be $144 \mathrm{~ns}$, or 12 clock cycles.

The device achieves the ultimate granularity using the NOP approach. By incrementally adding NOP instructions between two output changes, we observed prolong- ing of the pulse in steps of $12 \mathrm{~ns}$, or one clock cycle. Additionally, it is of interest to know the granularity of the delay loop approach. Instead of inferring this information in the same manner as in the case of the repeated NOP instructions, we perform a complete calibration of the dependence of delay duration on the number of delay loops.

The delay-loop calibration relies on measurements of the delay between two consecutive output changes for different numbers of delay loops, starting from one iteration and going up to the maximum number. The delay was measured using a time-to-digital converter (TDC, UQDevices) sensitive to rising edges only, so a two-pulse sequence was used for the measurements, the delay loop being placed between the pulses. To get the delay between the falling edge of the first pulse and the rising edge of the second one, and more specifically, the prolonging of the said delay with various increases of the number of delay-loop iterations, we took the TDC result for oneiteration delay loop as a reference, and subtracted it from the results for the other numbers of iterations. The final result yields the calibration curve showing linear dependence for delay loops, which tells us by what amount is the delay between two output changes prolonged if the number of iterations of the corresponding delay loop is increased from one to a certain amount, see Fig. 2.

The whole calibration procedure was repeated 9 times to verify the stability and the linearity of the delay-loop approach. For each loop-iteration increment we evaluated a min-max span of the corresponding delay duration across all the measurements, which characterizes the runto-run uncertainty of the given delay, see Fig. 3. In our case, the uncertainty is of the order of 100 ps for delays up to $100 \mathrm{~ms}$ and increases rapidly for larger delays.

To verify the perfect linear dependence, the acquired data were fitted to a linear function $y=a \times x$. From the slope of the linear fit, we estimated the delay-loop timing granularity to be $60 \mathrm{~ns}$, or 5 clock cycles. A mismatch between individual calibrations and the linear fit shows similar features as the run-to-run uncertainty, see Fig. 3 . We will show later that the increase of the uncertainty as well as of the mismatch is caused by internal clock drift and can be entirely removed using external clock source.

The maximum time interval between output changes was obtained from the calibration data for the delay loop with the highest possible number of iterations, with the resulting value of $255.7 \mathrm{~s}$.

The inter-channel simultaneity of output changes has also been studied. When all pulsebox channels were set to perform the change from a low voltage level to a high voltage level, the time difference between the rising edges at $50 \%$ signal level was found to be 2 ns at maximum between the slowest and the fastest channel. This value represents an upper bound on the simultaneity including all the inter-channel delays originated in the pulsebox, cables, and connectors.

The trigger latency has been measured by running a single-pulse sequence in the triggered mode of operation. A signal from an external generator is fed to the pulsebox. A copy of the signal is used to trigger an oscilloscope. The trigger latency is the time interval between the $50 \%$ crossings of the trigger signal and the first pulse of the 
pulsebox output. With the device in its current form, the trigger latency is around $900 \mathrm{~ns}$, with roughly $60 \mathrm{~ns}$ peak-to-peak jitter. These values are more than sufficient taking into account that the pulsebox is typically triggered by external events with periods much larger than $1 \mu s$, for example to synchronize the pulsebox with the power line frequency or with data acquisition. Once triggered, the output changes at all channels within a single sequence are generated with negligible sub-ns runto-run uncertainty and used to control all the circuitry and devices in the setup.

The maximum sequence complexity is characterized in terms of the maximum number of output changes and delay loops that can occur in a sequence. With rising complexity, the MCU program memory gradually fills up, until its capacity is exceeded. The maximum complexity was estimated using a model pulse sequence which uses a delay loop between each two consecutive output changes. For our current implementation the most complex sequence of this kind that we were able to generate consisted of 10670 output changes and 10669 delay loops.

\section{OPERATION WITH EXTERNAL CLOCK SOURCE}

By default, the Arduino Due development board runs with $84 \mathrm{MHz}$ master clock frequency, which is obtained from a $12 \mathrm{MHz}$ crystal oscillator using clock dividers and multipliers. Replacing this crystal with an external clock makes it possible to synchronize the operation of the pulsebox with other devices used in the experimental setup and eliminate the drift of the internal clock. Instead of the common $10 \mathrm{MHz}$ external clock we used a $12 \mathrm{MHz}$ one to facilitate the comparison of the pulsebox timing properties with the internal clock.

The calibration procedure has been repeated 8 times for the pulsebox with the external clock source, and indeed it shows a sub-nanosecond run-to-run uncertainty over the entire range of delays, see Fig. 4. The fit mismatch was also reduced below one nanosecond for all the acquired measurements and all possible delays of the pulsebox.

\section{POSSIBLE EXTENSIONS}

The functionality of the device can be further extended and some of the characteristics can be improved. As for the number of digital output channels, it is possible to go beyond 32. However, this means that more digital pin ports must be used and that multiple registers will sometimes have to be modified in order to perform an output change. This automatically leads to worse inter-channel output change simultaneity, as multiple corresponding registers cannot be changed using a single instruction. This can, however, be compensated in post-processing using a digital delay. The upper bound on the distance between two consecutive output changes can be overcome by either performing multiple delay loops or by nesting a delay loop inside another loop. The nested loop approach would yield a substantial increase of the range of

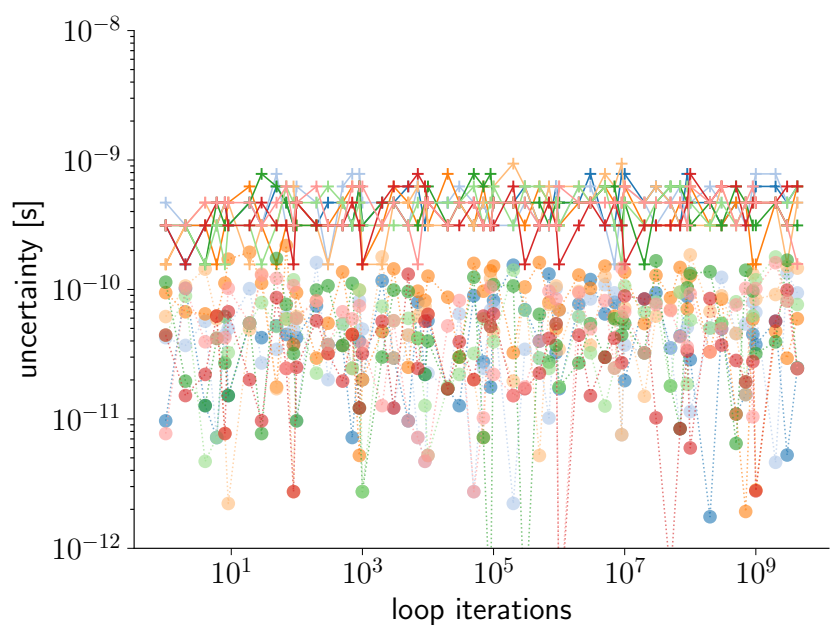

Figure 4. Calibration results for pulsebox operating with an external clock source. The delay duration span (cross marks, solid lines) and fit mismatch (circle marks, dotted lines) are presented, demonstrating perfectly stable operation with subnanosecond jitter.

possible delays.

Using an external clock source opens up the possibility of overclocked device operation. Higher clock rate allows for shorter pulses and lower granularity. The datasheet of the used MCU specifies the external oscillator frequency to be in the range of 3-20 MHz. This frequency is, by default, effectively multiplied by a factor of 7 . Overclocked, and even underclocked, operation has been achieved in our tests. However, we have observed that uploading code to the device fails for some frequencies of the external clock source. This can be avoided by changing the clock frequency to the $12 \mathrm{MHz}$ default for the upload and manipulating the clock frequency only afterwards. Furthermore, the operation stopped if the master clock frequency was too high. The master clock frequency can be further manipulated by changing the quotients of the built-in frequency dividers and multipliers, which will be subject to further tests. Alternatively, a faster-clock supporting MCU can be used to reach even better granularity.

The presented pulsebox generates unipolar signals. Bipolar operation can be attained using a two-way combiner to merge two unipolar channels into a single bipolar one, which was demonstrated for pulse generators by Strachan et al. and Haylock et al! ${ }^{[1213}$.

Finally, some of the channels could be used to feed programmable attenuators/amplifiers, direct digital synthesizer, or digital-to-analog converters. This would enable radio-frequency or arbitrary analog outputs. The performance of these outputs, such as the minimum distance between two signal changes, would depend on the parameters of the employed extension device. We tested interfacing the pulsebox to programmable digital attenuator operating from $9 \mathrm{kHz}$ to $6 \mathrm{GHz}$ (Mini Circuits ZX76$31 \mathrm{R} 75 \mathrm{PP}+$ ). Using direct parallel programming, we can command the attenuator to set a new attenuation level by sending 7 bit digital word within one clock cycle of our pulsebox ${ }^{20}$. 


\section{CONCLUSION}

We have presented a general-purpose digital pulsebox capable of producing complex sequences of pulses both in rapid succession and with great temporal separation, which sets it apart from devices built for one specific application. We have implemented the pulsebox using a low-cost ARM microcontroller and demonstrated its wide functionality, considerable precision, and sub-nanosecond timing jitter. The pulsebox timing granularity is $12 \mathrm{~ns}$, or one clock cycle, achieved by using delay loops together with single-cycle no-operation instructions. The presented 24 ns (2 clock cycles) minimum interval between output changes is a significant improvement over many-cycle intervals presented by many previous works. The employed delay-loop approach allows for extensive delays up to 255 s between the output changes while preserves the high temporal resolution and prevents excessive memory usage. The information about the shape of the sequence is communicated to the pulsebox by storing a specific program code in the memory. This approach, combined with the program memory capacity available on the MCU employed, yields the maximum sequence complexity of more than 20,000 output changes and delays.

Apart from designing the pulsebox itself, a set of characteristics and the methods of their measurement, applicable to a broad spectrum of similar devices, has been devised. We believe that these basic characteristics will facilitate characterization and comparison of future multi-channel digital pulse sequence generators.

\section{ACKNOWLEDGMENTS}

This work was supported by the Czech Science Foundation (project 17-26143S). RH also acknowledges the support by the Palacky University (project IGA-PrF2017-008). We would like to thank M. Dudka, I. Straka,
P. Obšil, and L. Slodička for many fruitful discussions on the pulsebox concept and applications.

\section{REFERENCES}

${ }^{1}$ R. W. Brown, Y.-C. N. Cheng, E. M. Haacke, M. R. Thompson, and R. Venkatesan, Magnetic Resonance Imaging: Physical Principles and Sequence Design (John Wiley and Sons, 2014).

${ }^{2}$ H. Häffner, C. F.Roos, and R. Blatt, Phys. Rep. 469, 155 (2008).

${ }^{3}$ S. Debnath, N. M. Linke, C. Figgatt, K. A. Landsman, K. Wright, and C. Monroe, Nature 536, 63 (2016).

${ }^{4}$ D. Bonneau, M. Lobino, P. Jiang, C. M. Natarajan, M. G. Tanner, R. H. Hadfield, S. N. Dorenbos, V. Zwiller, M. G. Thompson, and J. L. O'Brien, Phys. Rev. Lett. 108, 053601 (2012).

${ }^{5}$ M. Gräfe, R. Heilmann, M. Lebugle, D. Guzman-Silva, A. PerezLeija, and A. Szameit, J. Opt. 18, 103002 (2016).

${ }^{6}$ O. Alibart, V. D'Auria, M. De Micheli, F. Doutre, F. Kaiser, L. Labonté, T. Lunghi, É. Picholle, and S. Tanzilli, J. Opt. 18, 104001 (2016).

${ }^{7}$ S. Handa, T. Domalain, and K. Kose, Rev. Sci. Instrum. 78, 084705 (2007).

${ }^{8}$ P. E. Gaskell, J. J. Thorn, S. Alba, and D. A. Steck, Rev. Sci. Instrum. 80, 115103 (2009).

${ }^{9}$ E. E. Eyler, Rev. Sci. Instrum. 82, 013105 (2011).

${ }^{10}$ L. Sun, J. J. Savory, and K. Warncke, Concepts Magn. Reson. 43, 100 (2013).

${ }^{11}$ T. Pruttivarasin and H. Katori, Rev. Sci. Instrum. 86, 115106 (2015).

${ }^{12}$ B. Haylock, F. Lenzini, S. Kasture, P. Fisher, E. W. Streed, and M. Lobino, Rev. Sci. Instrum. 87, 054709 (2016).

${ }^{13}$ J. P. Strachan, V. Chembrolu, X. W. Yu, T. Tyliszczak, and Y. Acremann, Rev. Sci. Inst. 78, 054703 (2007).

${ }^{14}$ L. D. Pyeatt, Modern Assembly Language Programming with the ARM Processor (Elsevier, 2016).

${ }^{15}$ ARM Limited, DDI0337H_cortex_m3_r2p1_trm (2010), revision r2p1.

${ }^{16}$ Atmel Corporation, Atmel-11057C-ATARM-SAM3X-SAM3ADatasheet_23-Mar-15 (2015).

${ }^{17}$ D. Nino, H. Wang, and J. N. Milstein, Eur. J. Phys. 35, 055009 (2014).

${ }^{18}$ K. Cox, G. Greve, J. Weiner, and J. Thompson, Phys. Rev. Lett. 116, 093602 (2016).

${ }^{19}$ D. Saunders, J. Munns, T. Champion, C. Qui, K. Kaczmarek, E. Poem, P. Ledingham, I. Walmsley, and J. Nunn, Phys. Rev. Lett. 116, 090501 (2016).

${ }^{20}$ I. Straka et al., in preparation (2017). 